\title{
A novel method of mobility-based clustering protocol in software defined sensor network
}

Buwen Cao ${ }^{1,2,3}$, Shuguang Deng ${ }^{1,2,3^{*}}$, Hua Qin ${ }^{1,2,3}$ and Yue Tan ${ }^{1,2,3}$

*Correspondence: cbwchj@126.com; 814356787@qq.com ${ }^{1}$ College of Information and Electronic Engineering, Hunan City University, Yiyang 413000, China Full list of author information is available at the end of the article

\begin{abstract}
The distributed clustering method is widely used to extend network lifetime in traditional wireless sensor networks. However, it is difficult to achieve the idea performance of the whole network, such as transmission rate, energy consumption, and control overhead, neglecting the global stability of the network. To tackle this problem, a centralized mobility-based clustering (CMBC) protocol is proposed in the software defined sensor network. The method of CMBC mainly consists of two aspects: first, $\mathrm{CMBC}$ clusters the nodes with the connection time between the mobile nodes (i.e., noncluster head nodes, non- $\mathrm{CH}$ ) and the cluster head $(\mathrm{CH})$ and establishes stable topological structures between the non- $\mathrm{CH}$ s and the $\mathrm{CH}$; second, when emergencies occur, the centralization management control center sends the configuration files to replace the $\mathrm{CH}$. Compared to the distributed network of MBC, WCRA and IMP-MECA protocol, the proposed method can be applied in scenarios with high-speed mobile nodes to improve the network performance in terms of transmission successful rate, average power consumption, and average control overhead.
\end{abstract}

Keywords: Software defined sensor network, Cluster, Node mobility, Centralization

\section{Introduction}

Wireless sensor networks (WSNs) are self-organized ad-hoc network capable of sensing, data processing, and forwarding different physical parameters to users using multi-hop communication. They offer a flexible, self-adaptable, and low-cost solution to a number of distributed monitoring applications, especially in places with limited accessibility [1]. WSN consists of numerous small-scale sensor nodes, which can sense different kinds of physical phenomena, such as temperature, noise, and network speed, which transfer the collected data to the base station through multi-hop wireless communication. However, because of the limitation of the complex environment, battery energy, and the capacity of processing, storage and communication, the topological structure of WSN often changes $[1,2]$, resulting in serious challenges to their application. Therefore, topological control mechanism has become an important research topic.

Since from last two decades, a number of clustering algorithm based topological characteristics were proposed to optimize the energy utilization and improve the routing execution for WSN. Bagci et al. [3] proposed a low-energy adaptive clustering hierarchy $(\mathrm{LEACH})$, which groups the neighbor nodes into cluster in the network according to the

(c) The Author(s), 2021. Open Access This article is licensed under a Creative Commons Attribution 4.0 International License, which permits use, sharing, adaptation, distribution and reproduction in any medium or format, as long as you give appropriate credit to the original author(s) and the source, provide a link to the Creative Commons licence, and indicate if changes were made. The images or other third party material in this article are included in the article's Creative Commons licence, unless indicated otherwise in a credit line to the material. If material is not included in the article's Creative Commons licence and your intended use is not permitted by statutory regulation or exceeds the permitted use, you will need to obtain permission directly from the copyright holder. To view a copy of this licence, visit http:// creativecommons.org/licenses/by/4.0/. 
strength of the received signal, and selects one node in the cluster as the cluster head $(\mathrm{CH})$. This $\mathrm{CH}$ is responsible for the coordination of other nodes in the cluster and the fusion and forwarding of data in the cluster. To balance the energy of each node, LEACH periodically selects the $\mathrm{CH}$. However, LEACH cannot guarantee the optimal distribution of $\mathrm{CH}$, which affects the network lifetime. In Ref. [4], the authors proposed a balanced clustering algorithm with distributed self-organization, which mainly considers the distance between nodes and the density distribution of nodes, the simulation results show that the algorithm can effectively balance the size of clusters and prolong the network lifetime. Begum et al. [5] proposed a modified load-balancing clustering algorithm, which can not only balance the load among the sensor nodes, but also select $\mathrm{CH}$ according to residual energy. In Ref. [6], authors analyzed the performance of three topological control algorithms, i.e., CDS Rule K, EECDS, and A3), which are derived from the theory of CDS. On this basis, five design criteria for improving the performance of algorithms of CDs topology discovery algorithm are defined and a new topology control algorithm called clique-base CDs (CCDs) discovery is proposed, which can achieve large network coverage with minimal overhead. Heidemann et al. [7] proposed an adaptive clustering algorithm on the basis of geographical location (geographical adaptive fidelity, GAF), which adopts the sleep mechanism to reduce energy consumption, and uses these methods of clustering by virtual cells and the load balancing mechanism of node state transition to extend the network lifetime. In Ref. [8], a distributed hybrid energyefficient clustering approach (hybrid energy-efficient distributed) was proposed to select iteratively the $\mathrm{CH}$ in a period and take the residual energy of nodes and the cost of intracluster communication as parameters. Cisse et al. [9] presented a novel distributed clustering algorithm named HWCA, which selects the $\mathrm{CH}$ on the basis of the mixed matrix integrating the neighbor nodes and the distance between the neighbor nodes and the base station. The simulation results show that this method improves the lifetime of the network and reduces the frame loss rate. In Ref. [10], authors proposed an energy efficient and safe weighted clustering algorithm, ES-WCA, which effectively integrates five criteria for behavior levels, cluster stability, distance between nodes and neighbors, residual energy of nodes, and the connectivity degree of nodes. The simulation experiments prove the effectiveness of the algorithm. On the basis of the energy level and the relative position between the nodes and the base stations, Kim et al. [11] suggested a novel method for selecting the $\mathrm{CH}$ according to the node level, the base station calculates the round of a $\mathrm{CH}$ in advance, which effectively reduces the energy to replace the $\mathrm{CH}$, which prolongs the network lifetime. In Ref. [12], the authors posited a self-load balanced clustering algorithm. The simulated experimental results show that the proposed method outperforms the other algorithms of GSTEB and GA-EBCDS. Based on LEACH [13], an improved clustering approach is proposed, simulated experimental results demonstrate the proposed method is better than LEACH. Singh et al. [14] presented a novel weighted clustering and routing algorithm (WCRA), which utilizes node mobility, nodes lingering vitality and distance to form stable clusters and $\mathrm{CH}$. The algorithm mainly consists of two phases: first, the weight of each node is calculated and exploited in process of $\mathrm{CH}$ selection; second, in routing phase, the shortest, stable and energy efficient path are used to minimize the energy usage, thereby improving the throughput with the similar weight factor in the clustering process. Simulation experiments proved the effectiveness 


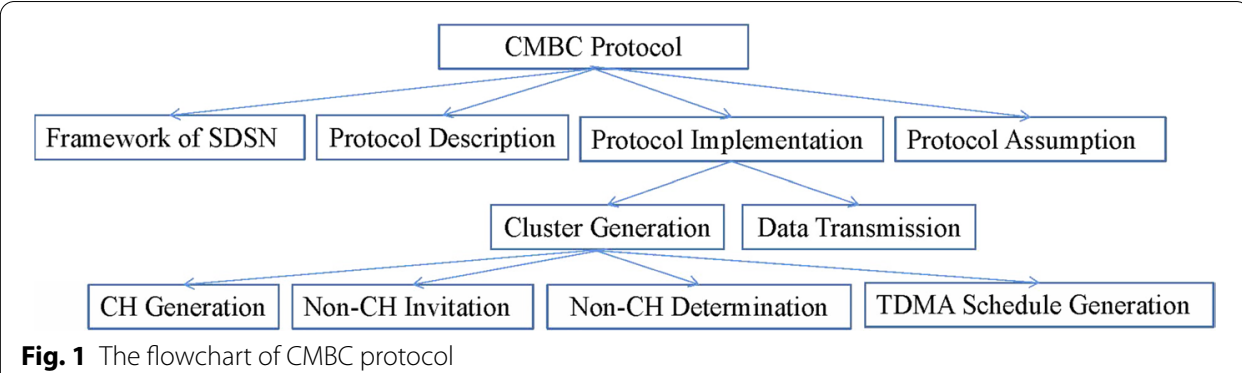

of the proposed method. Amini et al. [15] proposed an improved MECA model (i.e., IMP-MECA) that sensor nodes are randomly distributed across a hexagon, where the hexagonal is divided into equal cluster based on the radius of hexagonal. Simulation experiments showed the improvement of the residual energy between sensor nodes reduces total energy consumption, thereby improving the lifetime of wireless sensor networks related to previous models. Rachana et al. [16] proposed a method of mobile sink with mobile agent mobility model for WSN to study the effects of mobility on various performance parameters of the network and to explore the effective techniques to handle mobility in network. Experimental results showed that the proposed method can effectively improve the lifetime of the network.

The traditional network topology mainly extends the lifetime of WSN with the distributed clustering method [1]. However, nodes are randomly deployed in the monitoring area of distributed network, the mobility of node causes the dynamic change of the topological structure of wireless mobile sensor network. It is difficult to ensure the global stability of the network. Inspired by the clustering method in large-scale WSN [17-20], to overcome the above shortcomings, we propose a centralized mobility-based clustering $(\mathrm{CMBC})$ protocol in software defined sensor network (SDSN), which is a centralized wireless sensor network, which is described in Sect. 2.1. Compared to the distributed network of the MBC, WCRA and IMP-MECA protocol, our proposed method achieved the ideal performance in terms of transmission successful rate, average power consumption and average control overheads. CMBC can also be applied to high-speed mobile scenarios.

The contribution of this paper is mainly reflected in the following aspects: first, Considering the importance of clustering strategy in load balancing, a clustering protocol supporting node mobility is proposed in large-scale mobile wireless sensor network; second, to reduce the energy consumption in distributed mobile wireless senor network, we develop a mobility-based clustering protocol named as CMBC in centralized wireless sensor network, which is described as SDSN.

The remainder of this paper is organized as follows: Sect. 2 presents the methods used in this study and describes the CMBC protocol in detail. Section 3 shows the performance evaluation and the simulation results. Finally, we draw some conclusions. Figure 1 shows the flowchart of CMBC protocol. 


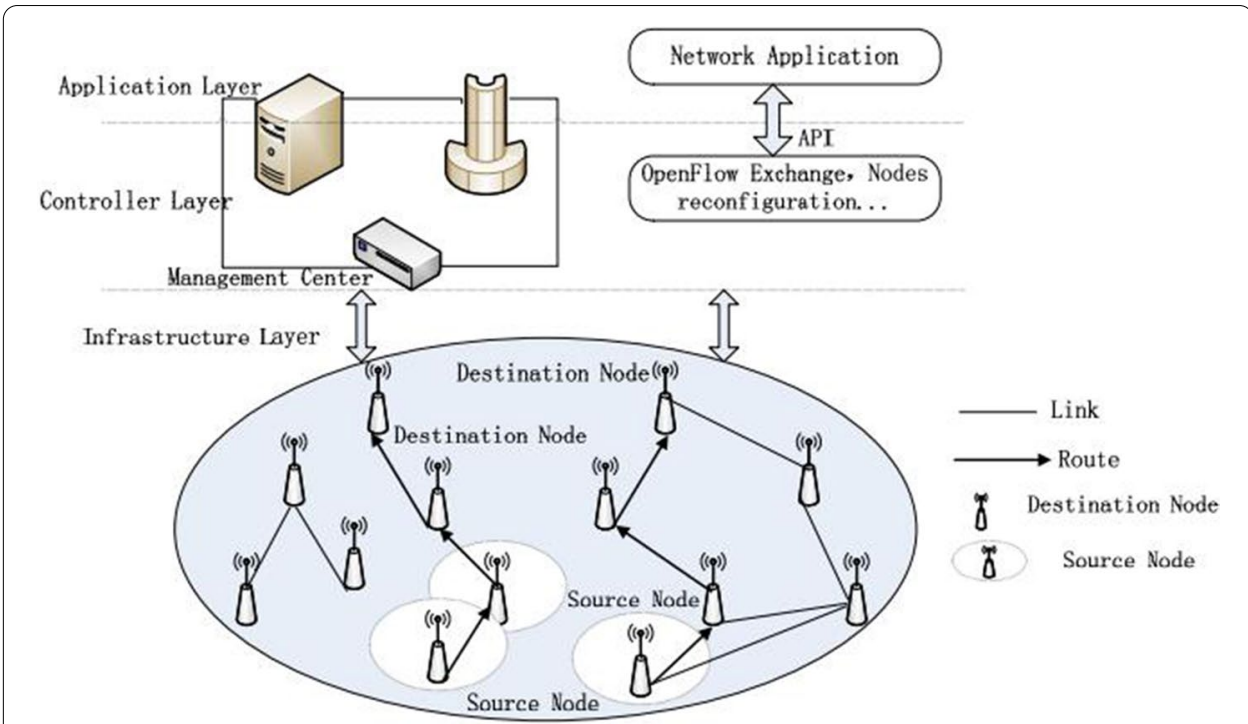

Fig. 2 The framework of SDSN

\section{Methods}

\subsection{The framework of SDSN}

Figure 2 shows the framework of the SDSN. Similar to software-defined network, SDSN can be divided into the infrastructure layer, control layer, and application layer from bottom to top. From Fig. 2, we can see clearly that the architecture of SDSN is essentially composed of a large number of software defined sensor nodes and centralized management control center (CMCC), of which the node structure is composed of sensor module and general programmable data transceiver unit, the CMCC configures uniformly the network control technologies such as network coverage, topology control and routing protocol, and distributes the corresponding configuration files to each node, which is to obtains the global optimal network performance. The main feature of this architecture is that each layer of the architecture can realize the reconfiguration of the sensor network according to the needs of users and the network state [1].

After deploying the network, the CMCC configures the sensing range of each sensing node in real time, so that the monitoring area is sensed and fully covered by SDSN.

\subsection{CMBC protocol description}

Inspired by the mobility-based clustering (MBC) protocol, the CMBC protocol is proposed to increase the control overhead and the power consumption caused by the request information sent to join the new cluster and avoid changes in the topological structure of the wireless mobile sensor network caused by the mobility of nodes. When the topology is changed due to the mobility of nodes, the management center re-clusters the entire network according to the information of nodes and builds effective and stable transmitting links. Figure 3 depicts the sequence structure of the CMBC protocol [18], where $p$ is the proportion of $\mathrm{CH}$ nodes in all nodes, $1 / p$ is the number of nodes in a cluster, and $\tau$ is the length of a time slot. 


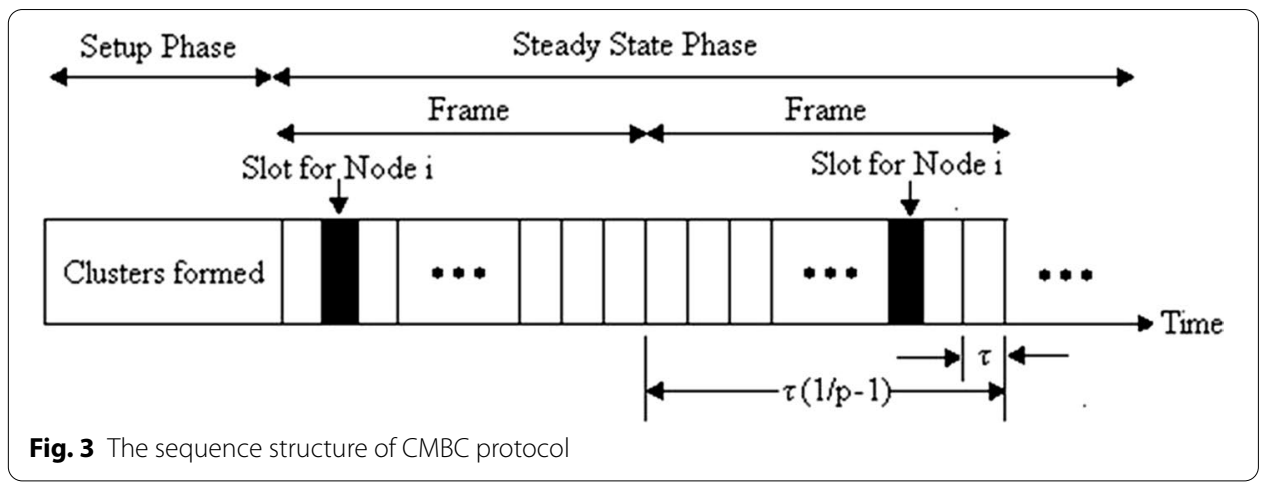

As shown in Fig. 3, similar to MBC protocol [18], the CMBC protocol is mainly divided into two stages. In the first stage, $\mathrm{CMBC}$ generates the cluster. To improve the success rate of the packet transmission, the non-cluster nodes (i.e., non- $\mathrm{CH}$ nodes) choose a node with strong stability as the $\mathrm{CH}$. In the second stage, $\mathrm{CMBC}$ transmits the data with stable transmitting links, and each non- $\mathrm{CH}$ node spontaneously transmits data to the $\mathrm{CH}$ according to the time division multiple access (TDMA) time series table formed by the cluster. When the connection time is exhausted, the non- $\mathrm{CH}$ nodes request information to find a suitable new cluster to reduce the packet loss rate. This new cluster is determined by the fitness value of the corresponding $\mathrm{CH}$. The fitness value shows the stability between the non- $\mathrm{CHs}$ and $\mathrm{CH}$. When the connection time between the non$\mathrm{CHs}$ and $\mathrm{CH}$ cannot guarantee the next round of data transmission, the $\mathrm{CH}$ deletes the information of the node from the TDMA schedule, sends it to the rest of the non-CHs, and reports it to the MCC. In contrast, the non-CHs request information to join the new cluster, and find a new $\mathrm{CH}$. The TDMA schedule of the cluster is re-justed and sent to all the non- $\mathrm{CHs}$ when a $\mathrm{CH}$ accepts the non- $\mathrm{CHs}$. In this process, we assume that the members in the cluster only interact with the $\mathrm{CH}$ without sending information to the MCC. This information is obtained by transmitting data among the $\mathrm{CH}$.

Figure $4 \mathrm{a}$ describes the process of node movement from an old cluster to a new cluster. This shows that Node 6 moves to Cluster J, whereas Node 9 moves away from Cluster J. Therefore, the TDMA schedule of Cluster J should also be modified accordingly, that is, Node 9 is deleted from the original TDMA schedule and is rearranged into a new TDMA schedule in ascending order of connection time $\Delta \mathrm{t}$ (Fig. 4b). The strategy of converting Node 8 to the time slot of the original Node 9 can improve not only the transmission success rate but also the effective utilization of channel bandwidth. In the $\mathrm{CMBC}$ protocol, each node can predict the time of maintaining a stable connection between itself and the $\mathrm{CH}$. Therefore, sending a request packet and a cluster member confirmation packet is unnecessary when sending data. This feature reduces the network control overhead and node energy. Algorithm 1 describes the execution process of non$\mathrm{CH}$ nodes (i.e., common nodes presented in Fig. 4a). The pseudocode of the CMBC protocol is presented in Algorithm 1 and 2. 


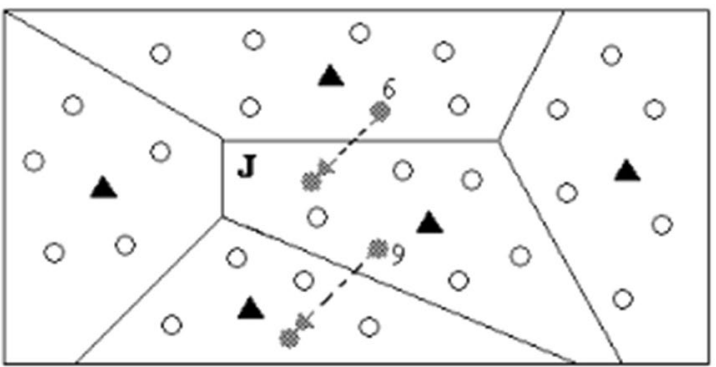

b

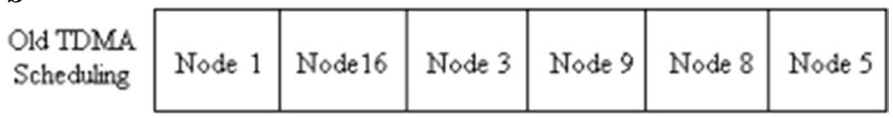

\begin{tabular}{|l|l|l|l|l|l|l|}
\hline $\begin{array}{c}\text { New TDMA } \\
\text { Scheduling }\end{array}$ & Node 1 & Node 16 & Node 3 & Node 8 & Node 6 & Node 5 \\
\hline
\end{tabular}

Fig. 4 The self-adaption sequence generation graph of CMBC

\section{Algorithm 1 The pseudocode of non-CH nodes}

1. While (sd=.T. )

2. \{ Adjust transmission power

3. Send data to $\mathrm{CH}$

4. Check joint time $\Delta \mathrm{t}$

5. if ( $\Delta \mathrm{t} \leq T_{\text {frame }} \| \mathrm{ACK}$ message is lost $)$ then

6. Send joint request message

7. elseif

8. Evaluate each new $\Delta \mathrm{t}$

9. Compute the value of each $\mathrm{CH}$ with the fitness value

10. Seek for a suitable $\mathrm{CH}$

11. Send a registration message to $\mathrm{CH}$

12. endif

13. endif

14. $\}$

After gathering in a cluster, non- $\mathrm{CHs}$ send the data to the $\mathrm{CMCC}$ through the $\mathrm{CH}$ and its neighboring $\mathrm{CH}$. The information, location, and range of these nodes are reported. During an emergency event, such as a $\mathrm{CH}$ runs out of energy or the connection time with non- $\mathrm{CHs}$ fails to continue to act as the $\mathrm{CH}$, the topological structure of the network is damaged, and the replacement request of $\mathrm{CH}$ is sent to the CMCC through its neighbor $\mathrm{CHs}$. After receiving the request, the $\mathrm{CMCC}$ responds 
and reconfigures the network resources in time and distributes the configuration files of the replacement of $\mathrm{CH}$ to the corresponding data-transmitting units. If the $\mathrm{CH}$ can continue to work, then the configuration files are discarded. Otherwise, the CMBC

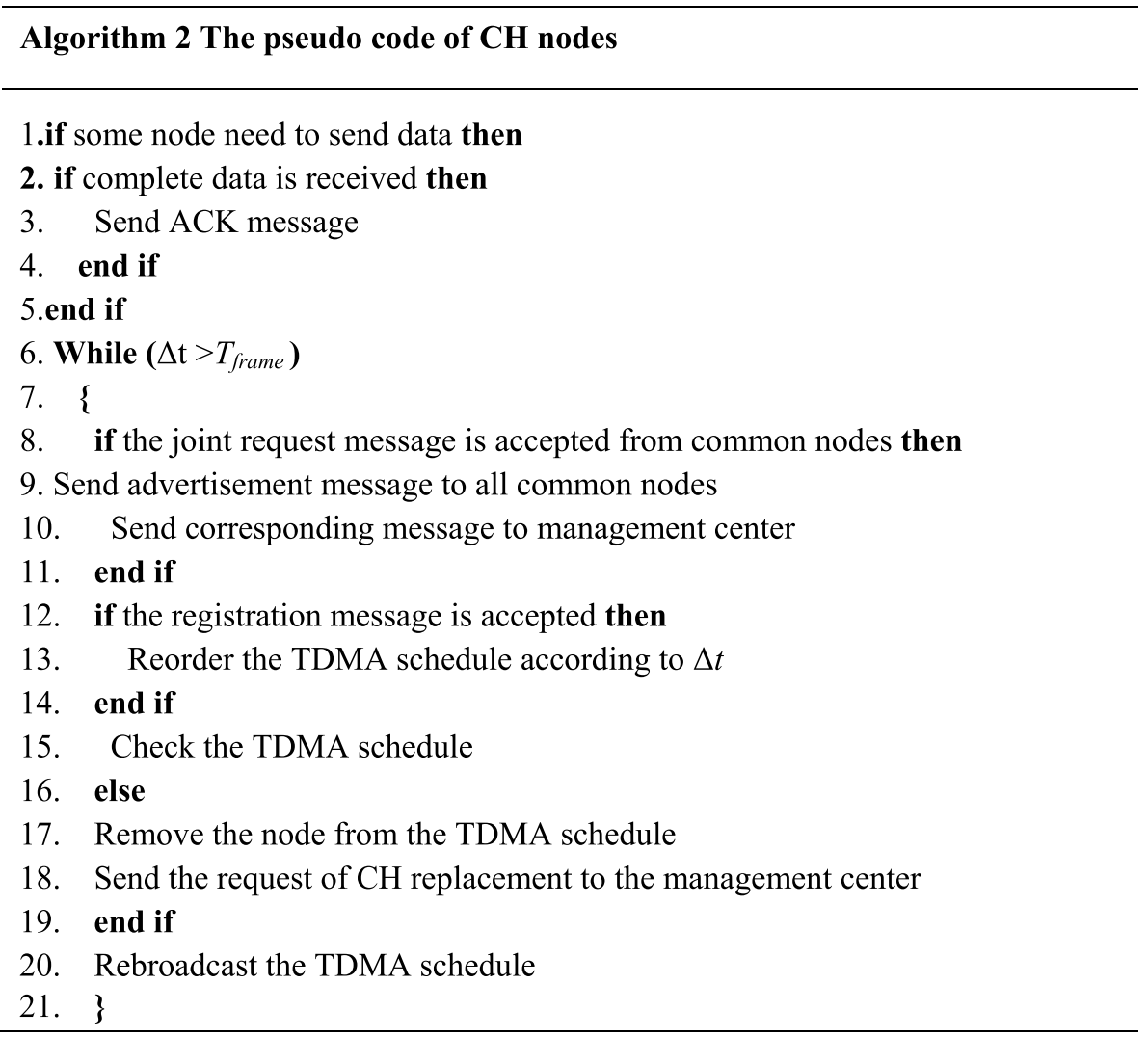

protocol is restarted.

\subsection{Protocol implementation}

Similar to LEACH-Mobile [3] and MBC [18], CMBC consists of the two stages of cluster generation and data transmission. The first stage mainly forms a series of clusters and creates the corresponding TDMA sequences. The second stage focuses on transmitting data between the $\mathrm{CHs}$ and the $\mathrm{CMCC}$.

\subsubsection{Cluster generation}

If a random number between $(0,1)$ generated by a sensor node is less than the threshold value $T(n)_{\mathrm{cls}}$, then the node is a $\mathrm{CH}$ [18]. $T(n)_{\mathrm{cls}}$ denotes the probability of being a $\mathrm{CH}$ and is determined by the correlation formula of the node residual energy and mobility. Each non- $\mathrm{CH}$ node joins the cluster where its $\mathrm{CH}$ can maintain the most stable connection. After determining the $\mathrm{CH}$, non- $\mathrm{CHs}$ send their registration information to the $\mathrm{CH}$. The $\mathrm{CH}$ receives the node as a member of the cluster and arranges a time slot for the node to transmit data and relevant information to the CMCC. The cluster generation largely consists of four sub-stages, namely, $\mathrm{CH}$ generation, non- $\mathrm{CH}$ invitation, non- $\mathrm{CH}$ determination, and TDMA schedule generation. 


\section{1. $\mathrm{CH}$ generation}

Prior to describing the process of $\mathrm{CH}$ generation, we give the description of the $\mathrm{CH}$. generation probability, which is described as follows [21]:

$$
T(n)_{\mathrm{cls}}=\frac{p}{1-p \times[r \bmod (1 / p)]}, \quad \forall n \in G,
$$

where $p$ is the ratio of the $\mathrm{CH}$ in the network. $1 / p$ is the node number in the cluster. $r$ is the round number generated by $\mathrm{CH}$. $\mathrm{G}$ is the node set that have not been selected as the $\mathrm{CH}$ in the nearest round of $r \bmod (1 / p)$. Equation (1) clearly shows that each node may be selected as $\mathrm{CH}$ in the round of $1 / p$. Notably, when $r=0$, the probability of each node to be selected as $\mathrm{CH}$ is $p$. In each round of $1 / p-1$, such nodes that have served as $\mathrm{CH}$ cannot continue to be $\mathrm{CH}$. After a round of $1 / p-1, T(n)_{\mathrm{cls}}=1$ means that all the remaining nodes have acted as $\mathrm{CH}$. In the beginning of a round of $1 / p$, the nodes in the network are selected as $\mathrm{CH}$ in the next round.

To prolong the network lifetime, the node with maximum residual energy is considered as the $\mathrm{CH}[3,18]$. Moreover, some researchers have proposed the selection of the node with the least relative mobility as the $\mathrm{CH}[22,23]$. To prevent low-energy nodes from becoming $\mathrm{CH}$, the $\mathrm{CMBC}$ protocol integrates the mobility rate and residual energy of the current node to achieve the energy balance of nodes in the network. In the CMBC protocol, the probability threshold of nodes acting as $\mathrm{CH}$ can be expressed as [21, 24]:

$$
T(n)_{\text {cls }}=\frac{p}{1-p \times[r \bmod (1 / p)]}\left(\frac{E_{n_{-} \text {curr }}}{E_{\max }} \times \frac{V_{\max }-V_{n_{-} \text {curr }}}{V_{\max }}\right), \quad \forall n \in G,
$$

where $E_{n_{-} \text {curr }}$ denotes the residual energy of the current node. $V_{n_{-} \text {curr }}$ means the mobility rate of the current node. $E_{\max }$ represents the original energy of the nodes in the network. $V_{\max }$ indicates the maximum mobility rate of nodes in the network. Equation (2) presents that the nodes with low mobility rate and high residual energy have a high probability of becoming a CH. $T(n)_{\mathrm{cls}}$ is normalized with $\frac{V_{\max }-V_{n} \text { curr }}{V_{\max }}$. Compared to high-speed movement, a $\mathrm{CH}$ should have lower speed, which can realize not only data transmission with CMCC but also a stable connection with cluster members. In the $\mathrm{MBC}$ protocol, $\mathrm{CH}$ has a fixed location relative to the base station. In the CMBC protocol, the position of $\mathrm{CH}$ can change along with time.

\section{Non- $\mathrm{CH}$ invitation}

When a node is selected as the $\mathrm{CH}$, it broadcasts an invitation message to the neighbor nodes and reports it to the CMCC. This information includes the location, speed, and direction of the $\mathrm{CH}$. The carrier sense multiple access/collision avoidance (CSMA/CA) MAC protocol is adopted to avoid collision during communication. To ensure that eligible nodes can receive messages, $\mathrm{CH}$ uses the maximum transmission power to broadcast the invitation information. At this stage, non-CHs must keep their receivers "ON" to receive the invitation information from $\mathrm{CH}$. 


\section{Non-CH determination}

Non-CHs may receive one or more invitation information from $\mathrm{CH}$, and the selection of a suitable $\mathrm{CH}$ should be selected by non-CHs. Similar to $\mathrm{MBC}$, the fitness of $W_{i j}$, which is related to the connection time, residual energy of the $\mathrm{CH}$, node degree of the $\mathrm{CH}$, and transmission distance, is utilized to represent the correlation between a non$\mathrm{CH}$ and the $\mathrm{CH}$ to which it is connected in this study. $W_{i j}$ is described as follows [21, 24]:

$$
W_{i j}=a \times \frac{E_{j \_c u r r}}{E_{\max } \times N_{j \_ \text {curr }}}+b \times\left(1-\frac{d_{i j}}{R_{\mathrm{tr}}}\right)+c \times \frac{\Delta t_{i j}}{t_{\text {frame }}},
$$

where $i$ is the non- $\mathrm{CH}$ node. $j$ is the $\mathrm{CH}$ node. $E_{j_{-}}$curr is the member number of the $\mathrm{CH}$ node $j . d_{i j}$ is the distance between the non- $\mathrm{CH}$ and $\mathrm{CH} . \Delta t_{i j}$ is the link time between the non- $\mathrm{CH}$ and $\mathrm{CH}, t_{\text {frame }}$ is the expected time to transmit a data frame. $R_{\mathrm{tr}}$ is the maximum transmission distance.

Equation (3) shows that $W_{i j}$ is a linear combination of $a, b$, and $c$, whose value depends on the importance of each factor and is expressed as follows [21, 24]:

$$
a+b+c=1
$$

where $a, b, \mathrm{c} \in(0,1) \cdot \frac{E_{j \_ \text {curr }}}{E_{\max } \times N_{j \text { curr }}}$ denotes the average normalized energy to each member node provided by $\mathrm{CH} j .1-\frac{d_{i j}}{R_{\mathrm{tr}}}$ represents the nodes that have large fitness values near the $\mathrm{CH}$ and realize their normalization. $N_{\exp }$ is the expected value of non- $\mathrm{CH}$, which can be expressed as follows [21, 24]:

$$
N_{\text {exp }}=\frac{N_{\text {total }}-p \times N_{\text {total }}}{p \times N_{\text {total }}}=\frac{1-p}{p},
$$

where $N_{\text {total }}$ denotes the total number of sensor nodes. $L_{\mathrm{p}}$ represents the length of the packet. $t_{\text {frame, }}$ which can be expressed as follows $[21,24]$ :

$$
t_{\text {frame }}=N_{\exp } \times \frac{L_{\mathrm{p}}}{R_{\mathrm{b}}}=\frac{1-p}{p} \times \frac{L_{\mathrm{p}}}{R_{\mathrm{b}}},
$$

where $R_{\mathrm{b}}$ denotes the data bit ratio, and $p$ refers to the probability of $\mathrm{CH}$ generation. Let $\tau=\frac{L_{\mathrm{p}}}{R_{\mathrm{b}}}$, Eq. (6) can be described as $[21,24]$

$$
t_{\text {frame }}=\tau \times\left(\frac{1}{p}-1\right)
$$

Equation (3) shows that the first factor considers the load balance of $\mathrm{CH}$ nodes when being clustered. In addition, this factor attempts to ensure the balance of residual energy and members of each $\mathrm{CH}$. The second factor guarantees a short distance between non- $\mathrm{CH}$ and $\mathrm{CH}$. The last factor ensures sufficient connection times between non- $\mathrm{CH}$ and $\mathrm{CH}$. The fitness value of each $\mathrm{CH}$ that sends invitation information to all the sensor nodes is calculated by Eq. (3), and those nodes with the maximum fitness value is determined as the $\mathrm{CH}$. Furthermore, the nodes transmit registration information to the selected $\mathrm{CH}$ via the CSMA/CA MAC protocol. In the decision stage, the receivers of all $\mathrm{CH}$ must keep "ON." 


\section{TDMA schedule generation}

After receiving the registration information from the cluster members, the $\mathrm{CH}$ forms the TDMA schedule in ascending order with the expected connection time of each cluster member. Each cluster member will sends information to the $\mathrm{CH}$ according to the specified time slot in the data transmission stage. Assume that $k$ is the frame number of data transmission, $0 \leq k \leq n$. $N_{i}$ is the order of cluster member in the TDMA schedule.

Node $i$ begins transmitting data spontaneously from $\left(N_{i}+\left(\frac{1}{p}-1\right) \times k\right) \times \tau$ to $\left(N_{i}+\left(\frac{1}{p}-1\right) \times k\right) \times \tau \geq \Delta t_{i j}$. The cluster members are arranged to ensure successful data transmission between cluster members and $\mathrm{CH}$, in ascending order of $\Delta t_{i j}$ in the stage of TDMA schedule generation, namely, $k \rightarrow \max$.

\subsubsection{Data transmission}

In the $\mathrm{CMBC}$ protocol, we assume that all nodes maintain their time synchronization and enter the clustering stage at the same time. All the non-CHs send the same data to some $\mathrm{CH}$ and transmit the data spontaneously according to the time slot allocated by the TDMA schedule. The $\mathrm{CH}$ transmits the data to the CMCC through its neighboring $\mathrm{CH}$. To prolong network lifetime, we introduce the heuristic wake-up mechanism to save the energy of nodes [25].

First, each sensor node rouses itself before ending its time slot according to the TDMA schedule and returns to its sleep state entering their time slot. Second, each node adaptively adjusts its transmission power to transmit data according to the distance between itself and the $\mathrm{CH}$, which can be calculated with the node information in the received data packet. Finally, the $\mathrm{CH}$ must keep the "ON" state to receive data and maintain the communication between the $\mathrm{CH}$ and the CMCC.

In the $\mathrm{MBC}$ protocol, each non- $\mathrm{CH}$ spontaneously transmits the data to the $\mathrm{CH}$ according to the TDMA schedule, and the $\mathrm{CH}$ integrates these data and transmits them to the base station under the multi-hop mode. Aparting from the above functions of two-way communication between the cluster members and the $\mathrm{CH}$, the $\mathrm{CMBC}$ protocol have the following functions. First, it should transmit the integrated data to the CMCC in a multi-hop manner. Second, each $\mathrm{CH}$ transmits its location information, residual energy, and the frame connection time to the $\mathrm{CMCC}$, which ensures that the $\mathrm{CMCC}$ can distribute the configuration files of replacing $\mathrm{CH}$ in the concentration mode.

Notably, in the actual environment, these incidents cause the failure of node hardware and the speed or direction of the change of mobile nodes suddenly, thereby leading to packet loss. To avoid the occurrence of similar situations, acknowledgments (ACKs) are added to the data transmission, which ensure that non- $\mathrm{CHs}$ can realize successful data transmission. Accordingly, after receiving the packets, the $\mathrm{CH}$ returns the ACKs to the non-CHs. When the non-CHs receive the ACK signal sent by the $\mathrm{CH}$, it indicates that the packets have been successfully sent. Otherwise, the non-CHs broadcast a request signal to join a new cluster. The $\mathrm{CH}$ receiving the request will send the invitation information to non-CHs according to Sect. 2.3.1.

To ensure that the $\mathrm{CH}$ successfully transmits data, when $\mathrm{CH}$ sends data to the $\mathrm{CMCC}$, the ACKs are also needed to be added. After receiving the data packets, the CMCC returns the ACKs to the $\mathrm{CH}$. When the $\mathrm{CH}$ receives the ACK sent by the $\mathrm{CMCC}$, it 
indicates that the data has been successfully sent to the CMCC. Otherwise, the $\mathrm{CH}$ will send a request of replacing the $\mathrm{CH}$ to the $\mathrm{CMCC}$, which ensures that the $\mathrm{CMCC}$ can centrally distribute the configuration file of replacing $\mathrm{CH}$.

In $\mathrm{CMBC}$ protocol, similar to $\mathrm{MBC}$ protocol, each non- $\mathrm{CH}$ node joins the cluster where its $\mathrm{CH}$ can maintain the most stable connection through its neighbor nodes, we can infer the complexity is $O(n)$ from Algorithm 1. Assuming that there are $S$ neighbor nodes for each non- $\mathrm{CH}$, then the complexity is $\mathrm{O}(\mathrm{Sn})$. Prior to joining the cluster, to achieve the energy balance of nodes in the network, the CMBC protocol selects lowenergy nodes as $\mathrm{CH}$ by integrating the mobility rate and residual energy of the current node. From Line 10 in Algorithm 1, we can analyze that the complexity of generating $\mathrm{CH}$ is $O(n)$. From Algorithms 1 and 2, we can infer that the complexity of CMBC protocol is $O\left(n^{2}\right)$.

For each non-CH node, the stable connection is built through its neighbor nodes according to the TDMA Scheduling. From Fig. 4, we can analyze that the computational complexity of the self-adaption sequence generation graph mainly depends on some an algorithm. For the quick sort algorithm, its computational complexity is $O(\operatorname{Sn} \log n)$, where $S$ means the number of neighbor node. For each $\mathrm{CH}$ node, when the emergency event occurs, the replacement request is sent from $\mathrm{CH}$ to $\mathrm{CMCC}$, which responds and reconfigures seasonably the network resources. Accordingly, $\mathrm{CH}$ distributes the configuration files of the replacement of $\mathrm{CH}$ to the corresponding data-transmitting units, i.e., non-CHs. The computational complexity of Algorithm 2 is $O\left(n^{2}\right)$. Therefore, the computational complexity of $\mathrm{CMBC}$ is $\varphi^{2} \log \varphi$.

In short, the computational complexity of $\mathrm{CMBC}$ protocol is effective in polynomial time.

Notably, 1500 sensors are randomly deployed in the two-dimensional area, which is $2000 \times 2000 \mathrm{~m}^{2}$. According to Algorithm 1 and Algorithm 2, we can infer that the convergence speed of our proposed method is relatively slow, the possible reason is that non- $\mathrm{CHs}$ seeks for the suitable $\mathrm{CH}$ within a frame time, and if a frame time is ended, the request of $\mathrm{CH}$ replacement to $\mathrm{CMCC}$ is iteratively sent. It will spend a lot of time to guarantee the execution process of our proposed algorithm. Obviously, the convergence of $\mathrm{CMBC}$ will be further improved with the decrease of the size of network.

\subsection{Protocol assumption}

In this study, the following assumptions are considered for analyzing the proposed protocol:

1. The CMCC is deployed at a fixed position beyond the network deployment area.

2. All sensors are randomly deployed in the two-dimensional area. These sensors know their position, moving speed, and direction.

3. Two sensors cannot be placed in the same position.

4. All sensors can accurately estimate the transmission time of data packets.

5. All sensor nodes have time synchronization.

6. All sensor nodes are the same, that is, they have similar energy, coverage radius, transmission capacity, and physical characteristics. 
The location, moving speed, direction, and time synchronization mentioned in the hypothesis can be obtained through the location and synchronization method in the CMBC protocol [18].

\section{Performance evaluation}

\subsection{Performance evaluation parameters and environment}

To assess the performance of the $\mathrm{CMBC}$, the following parameters are used in this study: transmission success rate, average power consumption, and average control overhead, which are described as follows [21, 24]:

$$
\begin{aligned}
& R_{\mathrm{r}}=\frac{N_{\mathrm{r}}}{K}, K=N_{\mathrm{r}}+N_{\mathrm{l}}, \\
& E_{\text {avg }}=\frac{E_{\text {total }}}{N_{\mathrm{r}}}, \\
& C_{\text {avg }}=\frac{C_{\text {total }}}{N_{\mathrm{r}}}=\frac{N_{\text {ctrl }} \times L_{\text {ctrl }}}{N_{\mathrm{r}}},
\end{aligned}
$$

where $N_{\mathrm{r}}$ is the number of received packets. $N_{\mathrm{l}}$ is the number of lost packets. $E_{\text {total }}$ is the total byte length of the control overhead. $N_{\text {ctrl }}$ is the number of control packets. $L_{\text {ctrl }}$ is the length of the control packet. The performance parameters of the CMBC protocol are listed in Table 1.

Table 1 show that the distance between non- $\mathrm{CHs}$ and $\mathrm{CH}$ is set within a range of 20-80 m. In the experiment, the transmission distance between $\mathrm{CH}$ and another $\mathrm{CH}$ and between $\mathrm{CH}$ and $\mathrm{CMCC}$ is allowed to exceed $80 \mathrm{~m}$. If the actual distance between non- $\mathrm{CHs}$ and $\mathrm{CH}$ is less than $0 \mathrm{~m}$, then the distance is set to $20 \mathrm{~m}$. If the actual distance is between 20 and $80 \mathrm{~m}$, then the transmission power consumption of the sensor node is computed with the actual distance. The results show that the average power consumption between $\mathrm{CH}$ and non- $\mathrm{CHs}$ decreases with the increasement in the percentage of cluster heads in the network [18]. For convenience, the ratio of $\mathrm{CH}$ is selected at $5 \%$ [18]. The experiments show that the $\mathrm{MBC}$ protocol achieves satisfactory results in the distributed sensor network [18]. In this study, we compare the CMBC, MBC, WCRA, and IMP-MECA protocols with the same experimental parameters.

Table 1 The parameter of CMBC protocol

\begin{tabular}{lc}
\hline Parameter & Value \\
\hline Network size & $2000 \times 2000 \mathrm{~m}^{2}$ \\
Sensor number & 1500 \\
Node deployment model & Two dimensional \\
& uniform distribu- \\
& tion \\
Data packet length & $2000 \mathrm{bit}$ \\
Control packet length & $100 \mathrm{bit}$ \\
Bit ratio & $9600 \mathrm{bps}$ \\
Transmission range within cluster & $20-80 \mathrm{~m}$ \\
\hline
\end{tabular}




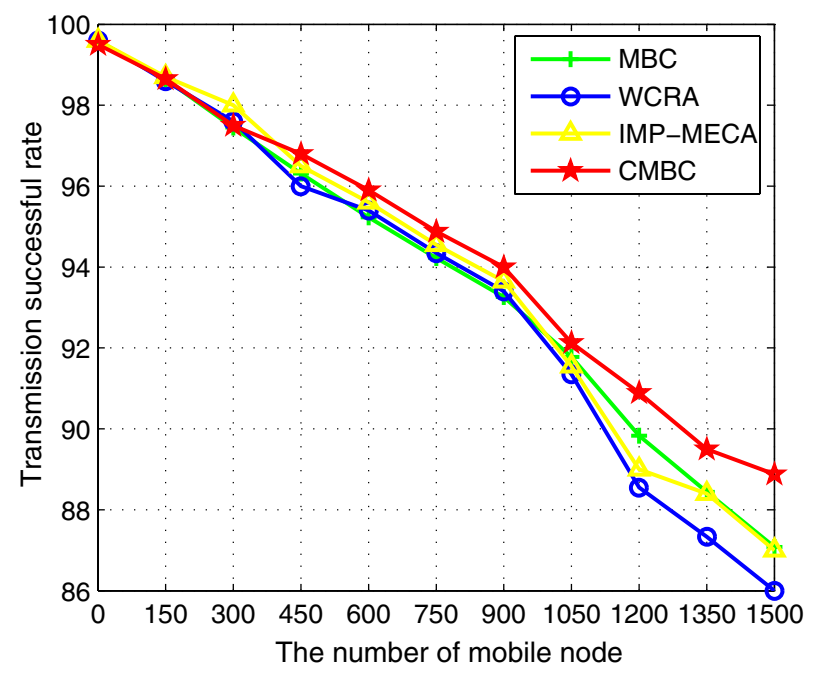

Fig. 5 Results comparison between the number of mobile node and the transmission success rate

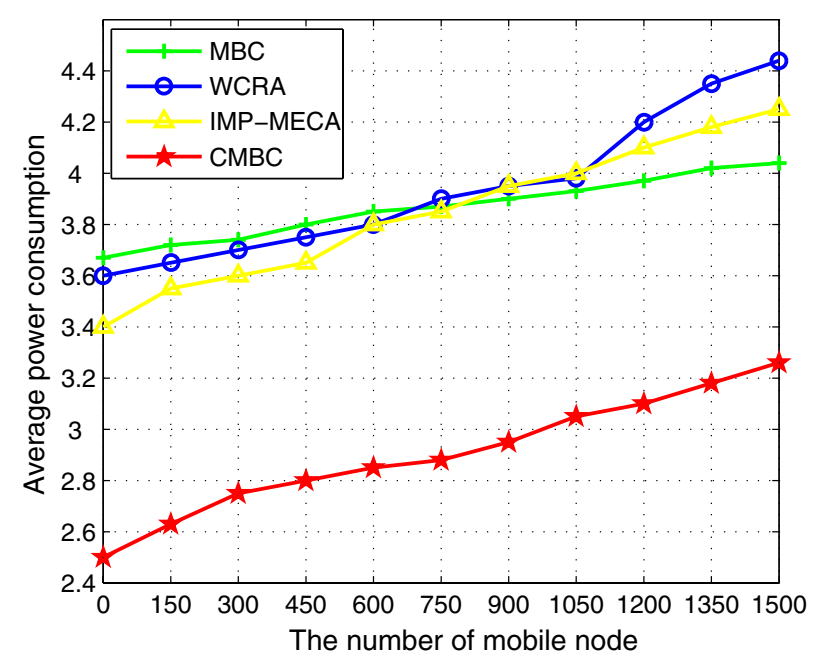

Fig. 6 Results comparison between the number of mobile node and the average power consumption

\subsection{Simulation results}

To compare the experiment result of centralized wireless sensor network and distributed wireless sensor network, the general random walk mobility model $[26,27]$ is introduced to model the mobility pattern of each node.

Figures 5, 6 and 7 present the transmission success rate, average power consumption, and average control overhead of the CMBC MBC, WCRA, and IMP-MECA protocols, respectively, when the maximum mobile rate is set to $25 \mathrm{~m} / \mathrm{s}$ [18]. The number of mobile nodes varies from 0 to 1500 with a 150 increment in large-scale SDSN.

Figure 5 shows the transmission success rate of a packet received by the four protocols when the number of mobile nodes changes. As shown in Fig. 5, when the number of mobile node is 0 , the transmission successful rate obtains the maximum value, it 


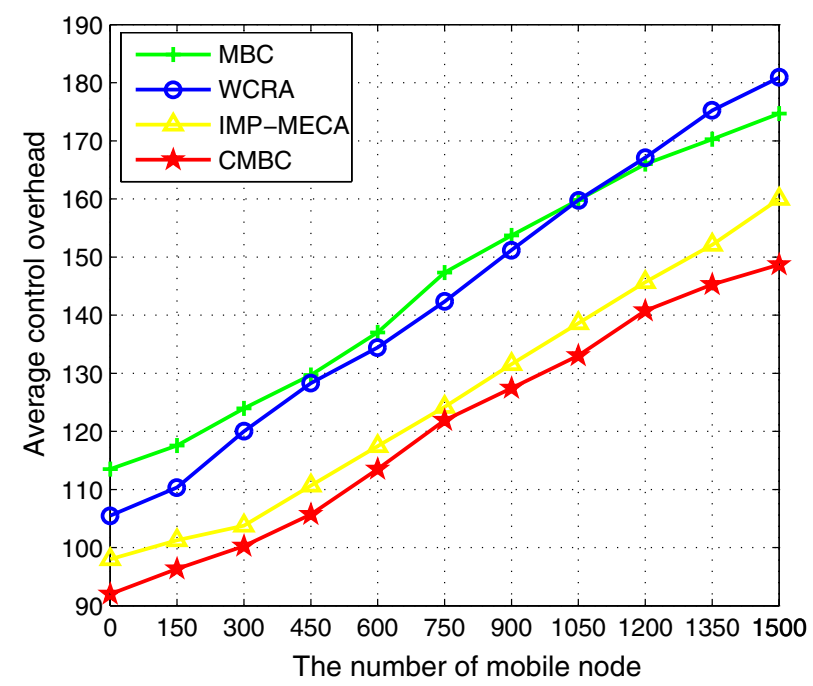

Fig. 7 Results comparison between the number of mobile node and the average control overhead

is because that all sensor nodes are in a static state, the connection link is in a stable transmission state, the data packets will be successfully transmitted. With the gradual increase of the nodes, the transmission success rate of four methods slowly decreases mainly due to the probability of a link break increases gradually with the increasement of mobile nodes. Notably, when the number of mobile node is less than 300, IMP-MECA achieved a high transmission success rate, it is because that IMP-MECA is designed for the medium scale wireless sensor networks, which the number of mobile nodes is no more than 300. The value of transmission success rate of WCRA is slightly superior to $C M B C$, the possible reason is that WCRA mainly is proposed to solve the problem of node mobility in small-scale networks, the number of mobile nodes is no more than 100. Compared to the other three protocols, the transmission success rate of the $\mathrm{CMBC}$ protocol slightly decreases because of the CMCC real time monitors the link state, which reduces the failure of the data transmission when certain links are damaged. This feature shows that the $\mathrm{CMBC}$ protocol is suitable for the larger scale mobile sensor networks.

Figure 6 describes the average power consumption of a packet received by the four protocols. With the addition of mobile nodes, the power consumption of the two protocols gradually increases because the non- $\mathrm{CH}$ nodes in the network consume additional power in the process of finding new cluster. This figure clearly shows that the power consumption of the CMBC protocol is significantly lower than that of the other three protocols when the number of mobile node changes mainly because the CMCC uniformly sends configuration files in the process of finding new cluster and effectively reduces the power consumption between the non- $\mathrm{CHs}$ and the $\mathrm{CH}$. Notably, when the number of mobile node is 0 , the average power consumption obtains the minimum value, it is because that all sensor nodes are in a static state, the connection link is in a stable transmission state, which needs not consume too much energy to deal with the mobile nodes.

Figure 7 presents the average control overhead of a packet received by the four protocols. With the increasement of mobile nodes, the average control overhead of the 
four protocols increases correspondingly, which is caused by the addition of unstable connections between the non- $\mathrm{CHs}$ and the $\mathrm{CH}$ in the cluster. Figure 7 illustrates that the average control overhead of the $\mathrm{CMBC}$ protocol is significantly lower than that of the other three protocols. When unstable connection occurs, the SDSN is uniformly deployed through the CMCC to reduce the time of the unstable connection. This reduction significantly improves the transmission success rate of the packet and reduces the average control overhead of the packet. Similar to Fig. 6, when the number of mobile node is 0 , the average control overhead achieves the minimum value, it is because that network protocols need not consume too much control overhead to deal with the mobile nodes.

Figure 8 demonstrates the changes of the transmission successful rate of the four protocols when the speed of mobile nodes changes from 5 to $40 \mathrm{~m} / \mathrm{s}$. With the increasement of the speed of mobile nodes, the connection stability between non$\mathrm{CHs}$ and $\mathrm{CH}$ is slowly weakened, which decreases the transmission successful rate of data packets. Interestingly, when the speed of mobile node is $5 \mathrm{~m} / \mathrm{s}$, which is approximately in static state, the connection link is in a stable transmission state, all data packets can be successfully transmitted, then the maximum value of transmission successful rate is achieved. When the speed of mobile nodes is less than $10 \mathrm{~m} / \mathrm{s}$, IMP-MECA achieves the maximum value of the transmission successful rate, WCRA obtains the second high value of the transmission successful rate. When the speed of mobile nodes is $15 \mathrm{~m} / \mathrm{s}$, IMP-MECA obtains the same second high value as CMBC, and WCRA obtains the high value of the transmission successful rate.

In Fig. 8, the curve of CMBC and MBC decreases slowly, while that of IMP-MECA and WCRA changes significantly, the transmission successful rate of IMP-MECA and WCRA is superior than that of CMBC when the speed of mobile node is less than $20 \mathrm{~m} / \mathrm{s}$, the possible reason is that the two methods are mainly designed to address the mobility for small scale wireless sensor network, with the increasement of the speed of mobile nodes, as shown in Fig. 8, when the speed of mobile node is greater than $20 \mathrm{~m} / \mathrm{s}$, the lifetime of wireless sensor networks is slowly weakened, thereby

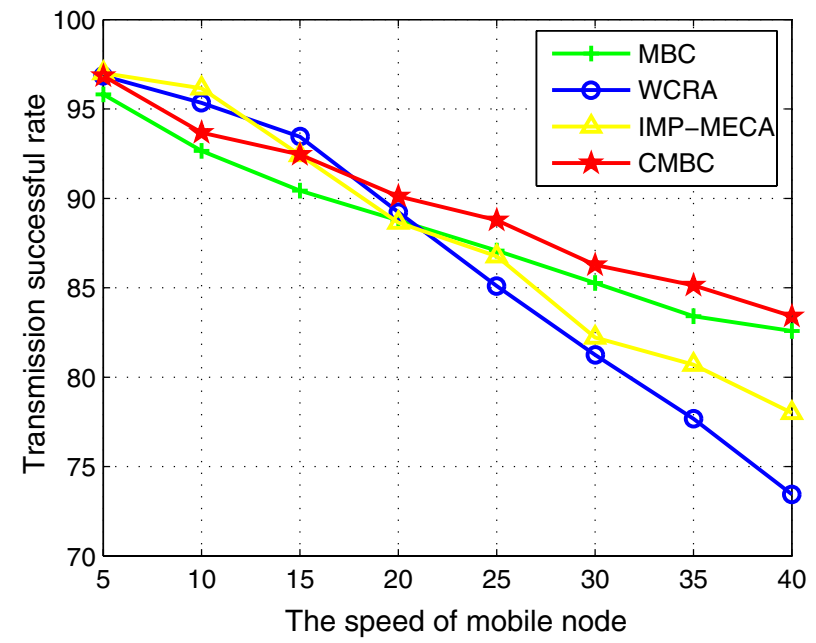

Fig. 8 Results comparison between the speed of mobile node and the transmission success rate 
decreasing the transmission successful rate. Accordingly, $\mathrm{CMBC}$ shows the surprising advantages. It is proved that $\mathrm{CMBC}$ is more suitable for large scale networks.

Figure 9 describes the comparison between the speed of mobile node and the average power consumption, when the speed of mobile node is $5 \mathrm{~m} / \mathrm{s}$, which is approximately in static state, the connection link is in a stable transmission state, the data packets can be successfully transmitted, $C M B C$ achieves the minimum value of average power consumption. With the addition of the speed of mobile node, the average power consumption is gradually increasing, to ensure the successful transmission of data packets, the number of new clusters added by the nodes from the broken link increases, the average energy consumption of packets increases slowly. As shown in Fig. 9, the performance of $\mathrm{CMBC}$ protocol is significantly better than that of the other three protocols. This improved performance effectively illustrates the effectiveness of the proposed clustering method based on node mobility in SDSN.

Figure 10 gives the results comparison between the speed of mobile node and the average control overhead. When the speed of mobile node is $5 \mathrm{~m} / \mathrm{s}$, which is approximately in static state, the connection link is in a stable transmission state, the data packets can be successfully transmitted, it is need not consume too much energy to deal with the mobile nodes, so the minimum value of average control overhead is achieved. With the addition of the speed of mobile node, the average control overhead is gradually increasing, to ensure the successful transmission of data packets, the number of new clusters added by the nodes from the broken link increases, leading to the increase in the average control overhead of packets. Notably, in Fig. 10, the average control overhead of all methods is slightly difference. From Figs. 9 and 10, we can clearly analyze that the CMCC uniformly sends configuration files in the process of node movement with the increasement of the speed of mobile node and effectively reduces the power consumption and the average control overhead between the non- $\mathrm{CH}$ s and the $\mathrm{CH}$, it is different from distributed wireless sensor network, which takes much longer to find new clusters.

Interestingly, as shown in Figs. 9 and 10, when the mobile speed of nodes is $5 \mathrm{~m} / \mathrm{s}$, the $\mathrm{CMBC}$ protocol has a significantly lower average energy consumption and average

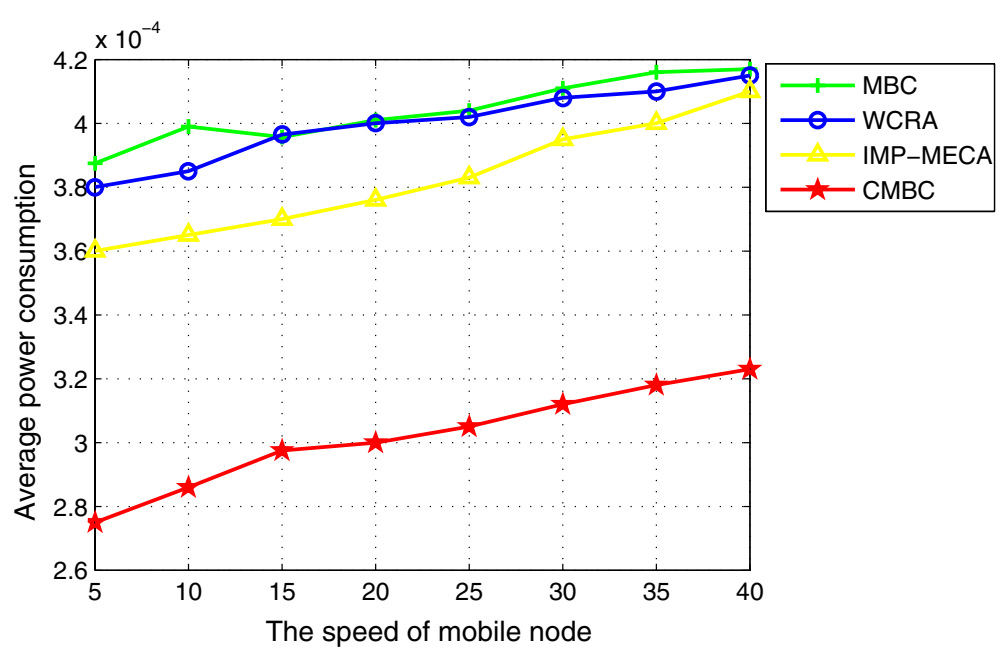

Fig. 9 Results comparison between the speed of mobile node and the average power consumption 


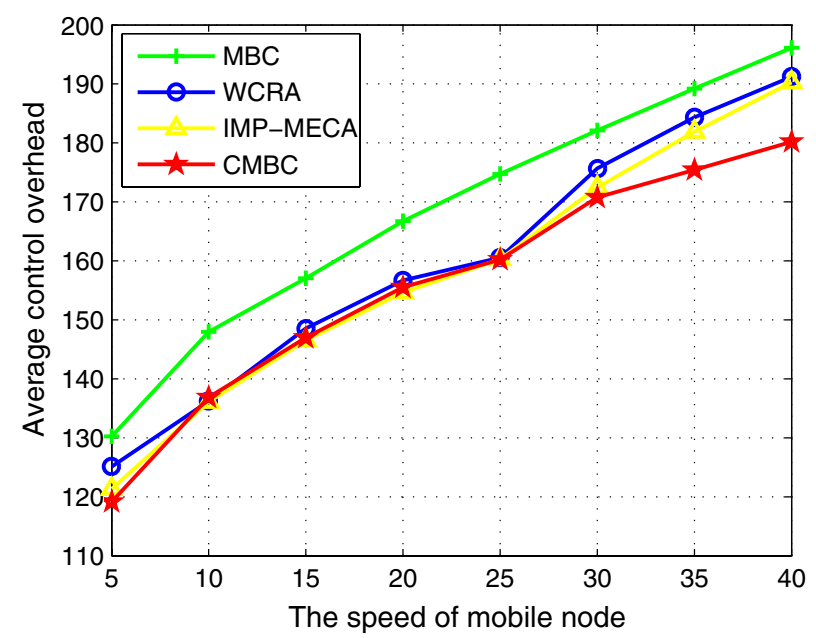

Fig. 10 Results comparison between the speed of mobile node and the average control overhead

control overhead than the other three protocols. It further demonstrates that the CMBC protocol also has satisfied network performance in the near-static environment.

\section{Conclusions}

Inspired by the $\mathrm{MBC}$ protocol, a centralized clustering protocol called $\mathrm{CMBC}$ based on node mobility is proposed to solve the shortcomings of routing failure caused by the dynamic topology change in distributed sensor networks. Our proposed protocol is implemented in SDSN when the data between non- $\mathrm{CHs}$ and $\mathrm{CH}$ are transmitted. Owing to energy exhaustion or the end of connection time with cluster nodes, they cannot continue to act as $\mathrm{CH}$, thus sending a request to replace the $\mathrm{CH}$ to the $\mathrm{CMCC}$ through neighboring $\mathrm{CH}$ nodes. Then, $\mathrm{CMCC}$ centrally reconfigures the network resources. Simulation results show that transmission success rate of the $\mathrm{CMBC}$ protocol is significantly higher than that of the MBC, WCRA and IMP-MECA protocol with lower average power consumption and control overhead. Compared to the distributed sensor network, the centralized sensor network of SDSN shows the significant performance.

For the future work, we'll further research the centralized wireless sensor network, the main work is as follows: (1) some important performance indicators, such as convergence rate and time, data transmission latency and the improvement of the centralized solution, etc. (2) network structure, such as network scale, larger transmission range, and the high dense wireless sensor network, etc. We also further research the theory proof for the experimental results.

\section{Abbreviations}

MBC: Mobility-based clustering; CMBC: Centralized mobility-based clustering; SDSN: Software defined sensor network; WSN: Wireless sensor network; CH: Cluster head; MCC: Management control center; CMCC: Centralization management control center; ACK: Acknowledgment; TDMA: Time division multiple access; MAC: Media access control.

\section{Acknowledgements}

This work was supported in part by the National Natural Science Foundation of China under Grants 61572180, 61472467, 61471164, 61672011, 61602164 and the Hunan Provincial Natural Science Foundation of China under Grants $2016 J J 2012$ and the Key Project of the Education Department of Hunan Province under Grant 20A087, Scientific Research Fund 
Education Department of Hunan Province of China under Grant 14b033 and the Innovation Platform Open Fund Project of Hunan Provincial Education Department Grant 20K025.

\section{Authors' contributions}

B.C. and S.D. are the principal contributors in terms of centralized mobility-based clustering modeling and the generation/interpretation of numerical results. In a supervising role, B.C. and S.D. formulated the research problem and contributed to the centralized mobility-based clustering modeling and the discussion of results. All authors read and approved the final manuscript.

\section{Availability of data and materials}

All data generated or analyzed during this study are included in this published article.

\section{Competing interests}

The authors declare that they have no competing interests.

\section{Author details}

${ }^{1}$ College of Information and Electronic Engineering, Hunan City University, Yiyang 413000, China. ${ }^{2}$ Key Laboratory of City Computing and IoT, Hunan City University, Yiyang 413000, China. ${ }^{3}$ Engineering Research Center of DongTing Lake Regional Ecological Environment Intelligent Monitoring and Disaster Prevention and Mitigation, Yiyang 413000, China.

Received: 31 January 2020 Accepted: 11 February 2021

Published online: 21 April 2021

\section{References}

1. A. Dahane, A. Loukil, B. Kechar et al., Energy efficient and safe weighted clustering algorithm for mobile wireless sensor networks. Mob. Inf. Syst. 34, 63-70 (2015)

2. C.P. Tang, S.K. Shokla, G. Modhawar et al., An effective collaborative mobile weighted clustering schemes for energy balancing in wireless sensor networks. Sensors 16(2), 261 (2016)

3. F. Bagci, Energy-efficient communication protocol for wireless sensor networks. Ad Hoc Sens. Wirel. Netw. 30(3-4), $301-322(2016)$

4. Y. Liao, H. Qi, W.Q. Li, Load-balanced clustering algorithm with distributed self-organization for wireless sensor networks. IEEE Sens. J. 13(5), 1498-1506 (2013)

5. S. Begum, R. Appusamy, Modified load-balanced clustering algorithm with distributed self-organization for wireless sensor networks. Int. J. Pharm. Technol. 8, 23349-23356 (2016)

6. H.K. Qureshi, S. Rizvi, M. Saleem et al., Evaluation and improvement of CDS-based topology control for wireless sensor networks. Wirel. Netw. 19(1), 31-46 (2013)

7. Y. Xu, H. Heidemann, D. Estrin, Geography-informed energy conservation for ad hoc routing, in Proceedings of the 7th Annual ACM/IEEE International Conference on Mobile Computing and Networking (2001)

8. O. Younis, S. Fahmy, HEED: a hybrid, energy-efficient, distributed clustering approach for ad hoc sensor networks IEEE Trans. Mob. Comput. 3(3), 366-379 (2004)

9. C.S.M. Cisse, A hybrid weight-based clustering algorithm for wireless sensor networks. Open Access Libr. J. 2(5), 1-10 (2015)

10. D. Amine, B. Nassreddine, K. Bouabdellah, Energy efficient and safe weighted clustering algorithm for mobile wireless sensor networks. Procedia Comput. Sci. 34, 63-70 (2014)

11. J.W. Kim, J.W. Kim, Energy efficient clustering algorithm for the mobility support in an IEEE 802.15 .4 based wireless sensor network. Wirel. Netw. 25(6), 3441-3452 (2019)

12. P.A.S. Chinnasamy, T.N. Janakiraman, Self-load balanced clustering algorithm for routing in wireless sensor network. Int. J. Intell. Syst. Appl. 9(9), 46-57 (2017)

13. S.P. Singh, S.C. Sharma, Improved clustering algorithm for wireless sensor network, in Soft Computing: Theories and Applications, ed. by M. Pant, K. Ray, T.K. Sharma, S. Rawat, A. Bandyopadhyay (Springer, Singapore, 2018), pp. 379-386

14. R.B. Lohani, V. Singh, Mobility aware energy efficient clustering for wireless sensor network, in 2019 IEEE International Conference on Electrical, Computer and Communication Technologies (ICECCT) (2019)

15. S.M. Amini, A. Karimi, S.R. Shehnepoor, Improving lifetime of wireless sensor network based on sinks mobility and clustering routing. Wirel. Pers. Commun. 109(3), 2011-2024 (2019)

16. R. Borawake-Satao, R.S. Prasad, Mobile sink with mobile agents: effective mobility scheme for wireless sensor network, in Sensor Technology: Concepts, ed. by M. Khosrow-Pour (Methodologies, Tools, and Applications (IGI Global, 2020), pp. 1035-1047

17. M. Masdari, S. Barshande, S. Ozdemir, CDABC: chaotic discrete artificial bee colony algorithm for multi-level clustering in large-scale WSNs. J. Supercomput. 75(11), 7174-7208 (2019)

18. S. Deng, J. Li, L. Shen, Mobility-based clustering protocol for wireless sensor networks with mobile nodes. IET Wirel. Sens. Syst. 1(1), 39-47 (2011)

19. P. Neamatollahi, M. Naghibzadeh, Distributed unequal clustering algorithm in large-scale wireless sensor networks using fuzzy logic. J. Supercomput. 74(6), 2329-2352 (2018)

20. N. Wang, D. Chen, J. Chen et al., Clustering data gathering method based on compressed sensing in wireless sensor networks, in 2018 10th International Conference on Intelligent Human-Machine Systems and Cybernetics (IHMSC) (2018)

21. W.B. Heinzelman, A.P. Chandrakasan, H. Balakrishnan, An application-specific protocol architecture for wireless microsensor networks. IEEE Trans. Wirel. Commun. 1(4), 660-670 (2002)

22. G. Renugadevi, M.G. Sumithra et al., An analysis on LEACH-mobile protocol for mobile wireless sensor networks. Int. J. Comput. Appl. 65(21), 38-42 (2013) 
23. M. Arshad, M.Y. Aalsalem, F.A. Siddqui, Energy efficient cluster based routing scheme for mobile wireless sensor networks, in 20145 th International Conference on Intelligent and Advanced Systems (ICIAS) (2014), pp. 195-206

24. J. Wang, L.B. Milstein, CDMA overlay situations for microcellular mobile communications. IEEE Trans. Commun. 43(2/3/4), 603-614 (2002)

25. A. Cerpa, D. Estrin, ASCENT: adaptive self-configuring sensor networks topologies, in Proceeding of 11 th Joint Conference on IEEE Computer and Communications Societies (2002), pp. 1278-1287

26. D.S. Kim, Y.J. Chung, Self-organization routing protocol supporting mobile nodes for wireless sensor network, in First International Multi-Symposiums on Computer and Computational Sciences (Imsccs 2006), Proceedings, vol. 2 (2006), p. 622

27. S.A.B. Awwad, C.K. Ng, N.K. Noordin et al., Cluster based routing protocol for mobile nodes in wireless sensor network, in Proceedings of the 2009 International Symposium on Collaborative Technologies and Systems (2009), pp. 233-241

\section{Publisher's Note}

Springer Nature remains neutral with regard to jurisdictional claims in published maps and institutional affiliations.

\section{Submit your manuscript to a SpringerOpen ${ }^{\circ}$ journal and benefit from:}

- Convenient online submission

- Rigorous peer review

Open access: articles freely available online

- High visibility within the field

- Retaining the copyright to your article

Submit your next manuscript at $>$ springeropen.com 